Article

\title{
Proximity Array Device: A Novel Photon Detector Working in Long Wavelengths
}

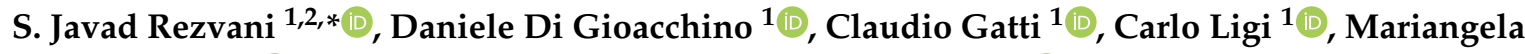 \\ Cestelli Guidi ${ }^{1}\left(\mathbb{0}\right.$, Sara Cibella ${ }^{3}$, Matteo Fretto ${ }^{2}$, Nicola Poccia ${ }^{4}\left(\mathbb{D}\right.$, Stefano Lupi ${ }^{5}$ and Augusto \\ Marcelli ${ }^{1,6}$ (1)
}

1 Istituto Nazionale di Fisica Nucleare, Laboratori Nazionali di Fisica Nucleare, Via Enrico Fermi 54, 00044 Frascati, Italy; daniele.digioacchino@lnf.infn.it (D.D.G.); claudio.gatti@lnf.infn.it (C.G.); carlo.ligi@lnf.infn.it (C.L.); mariangela.cestelliguidi@lnf.infn.it (M.C.G.); augusto.marcelli@lnf.infn.it (A.M.)

2 Istituto Nazionale di Ricerca Metrologica (INRiM), Strada delle Cacce 91, 10135 Torino, Italy; m.fretto@inrim.it

3 Consiglio Nazionale delle Ricerche, Istituto di Fotonica e Nanotecnologie, Rome 00156, Italy; sara.cibella@ifn.cnr.it

4 Institute for Metallic Materials, IFW-Dresden, Dresden 01069, Germany; n.poccia@ifw-dresden.de

5 INFN and Department of Physics, Università degli Studi di Sapienza, 00185 Rome, Italy; stefano.lupi@roma1.infn.it

6 International Centre for Material Science Superstripes, RICMASS, via dei Sabelli 119A, 00185 Rome, Italy

* Correspondence: rezvani@Inf.infn.it

Received: 7 April 2020; Accepted: 23 April 2020; Published: 1 May 2020

\begin{abstract}
We present here an innovative photon detector based on the proximity junction array device (PAD) working at long wavelengths. We show that the vortex dynamics in PAD undergoes a transition from a Mott insulator to a vortex metal state by application of an external magnetic field. The PAD also evidences a Josephson I-V characteristic with the external field dependent tunneling current. At high applied currents, we observe a dissipative regime in which the vortex dynamics is dominated by the quasi-particle contribution from the normal metal. The PAD has a relatively high photo-response even at frequencies below the expected characteristic frequency while, its superconducting properties such as the order parameter and the Josephson characteristic frequency can be modulated via external fields to widen the detection band. This device represents a promising and reliable candidate for new high-sensitivity long-wavelength detectors.
\end{abstract}

Keywords: niobium; proximity effects; superconductivity; detectors; terahertz; Vortex dynamics

\section{Introduction}

Long-wavelength radiation has recently become one of the most significant regions of the electromagnetic spectrum in terms of multi-disciplinary use not only in basic science research, but also in different technologies [1-3]. This wavelength range $\lambda=250-2500 \mu \mathrm{m}$ (energy range 0.5-4 $\mathrm{meV}$ ) allows the investigation of many fundamental physical phenomena, e.g., phonon and plasmon dynamics, elementary particle physics and probably in the near future even the cold dark matter [4-9]. Furthermore, with its high transmission through a wide range of non-conducting materials, long-wavelength radiation and in particular terahertz $(\mathrm{THz})$ radiation holds a significant potential in many applications such as non-destructive imaging, security screening and material characterizations. However, in comparison with adjacent energy ranges, e.g., IR and UV, in this wavelength domain the instrumentation and in particular the detectors are still limited, expensive or difficult to use. For instance semiconducting bolometers are widely used as cryogenic and non-cryogenic $\mathrm{THz}$ detectors [10], but their sensitivity to temperature and mechanical fluctuations and their limitations in the 
high frequency band operation, renders its use mainly confined to laboratories. Recent studies have shown that also superconducting devices can be employed for long wavelengths and in particular for $\mathrm{THz}$ generation and detection [11-14]. These devices exhibit extreme low noise compared to their semiconducting counterparts, while having response time orders of magnitude lower and a higher frequency range of operation. However, the frequency range of the operation is set by the superconducting gap of the material (usually in the range $300 \mathrm{GHz}$ to $1 \mathrm{THz}$ in conventional superconductors and up to $30 \mathrm{THz}$ in cuprates). Furthermore, there are studies showing the possibility to control semiconducting and superconducting properties in materials with physico-chemical treatments [15,16] and systems with low dimensionality [17-20]. Meanwhile, in recent years several distinctive mechanisms are being explored as new detection methods in this energy domain such as tunnel junctions, high gap superconducting junctions, transition edge sensors, bi-crystal junctions, etc. [21-23].

In this work we present and characterize a novel device based on the superconducting proximity junction array that can be used for detection of photons at long wavelengths. The superconducting characteristics of this device based on non-localized vortex dynamics will be discussed. The effect of the quasi-particle currents and the subsequent limitations of this detector will be also reviewed. The response of the device in the radio-frequency domain well below the expected characteristic frequency of the proximity junctions will be presented and further advancements based on non-equilibrium phenomena due to the external perturbations will be discussed. The proposed device represents a step forward toward the design of a robust, low-noise, broadband, high-sensitivity, and long-wavelength radiation detector operating in a wide energy range covering the $\mathrm{THz}$ domain.

\section{Experiment}

The device we designed and realized consists of an array of about $90000 \mathrm{Nb}$ superconducting islands regularly deposited on a non-superconducting substrate of $80 \times 80 \mu \mathrm{m}^{2}$ in size and with a period of $\sim 270 \mathrm{~nm}$, with an island diameter of $\sim 220 \mathrm{~nm}$ and a thickness of $45 \mathrm{~nm}$ (see Figure 1). Standard photo-lithographic tools have been employed to obtain a $40 \mathrm{~nm}$ thick Au template on the non-superconducting $\mathrm{Si} / \mathrm{SiO}_{2}$ substrate $\left(1 \times 1 \mathrm{~cm}^{2}\right)$. The template consists of a central square of $82 \times 82 \mu \mathrm{m}^{2}$, with the corners connected to four terminals used for electric measurements. The size of these terminals is $200 \times 200 \mu \mathrm{m}^{2}$, large enough for micro bonding. The $\mathrm{Nb}$ pattern is then created on top of the central Au square employing electron beam lithography and DC sputtering. To ensure a uniform current injection inside the array, two $45 \mathrm{~nm} \mathrm{Nb}$ bus bars were deposited along two opposite sides of the array. The IV measurements were carried out in a shielded cryostat at $4.2 \mathrm{~K}$. A current bias was applied using the signal generator at $107 \mathrm{~Hz}$. Two measurements mode were used. In one the I-V curves were measured amplifying the output voltage via a low-noise amplifier and digitized by the National instruments sampling card (USB-6363). In the second mode, the voltage sinusoidal waveform was measured via a lock-in amplifier locked to the applied current signal, resulting in the direct measurement of the dynamic resistance. The magnetic field was applied by placing a solenoid around the sample with the field perpendicular to the plane of the device. The current through the solenoid increased in a step-wise fashion and separate I-V traces were recorded at each field step.

For the radio-frequency response of the device, a custom-made diamagnetic dipole antenna with length $\mathrm{L}=\lambda / 2$, centered at $8 \mathrm{GHz}$ was inserted inside the cryostat in front of the magnetic coils and the device. The highest transmission line (resonant frequency) using a vector analyzer connected to the antenna was found at $f=7.78 \mathrm{GHz}$ at which the measurements were done. The response measurements were carried out via a continuous measurement of the electrical response of the device with and without the radio-frequency (RF) signal. 


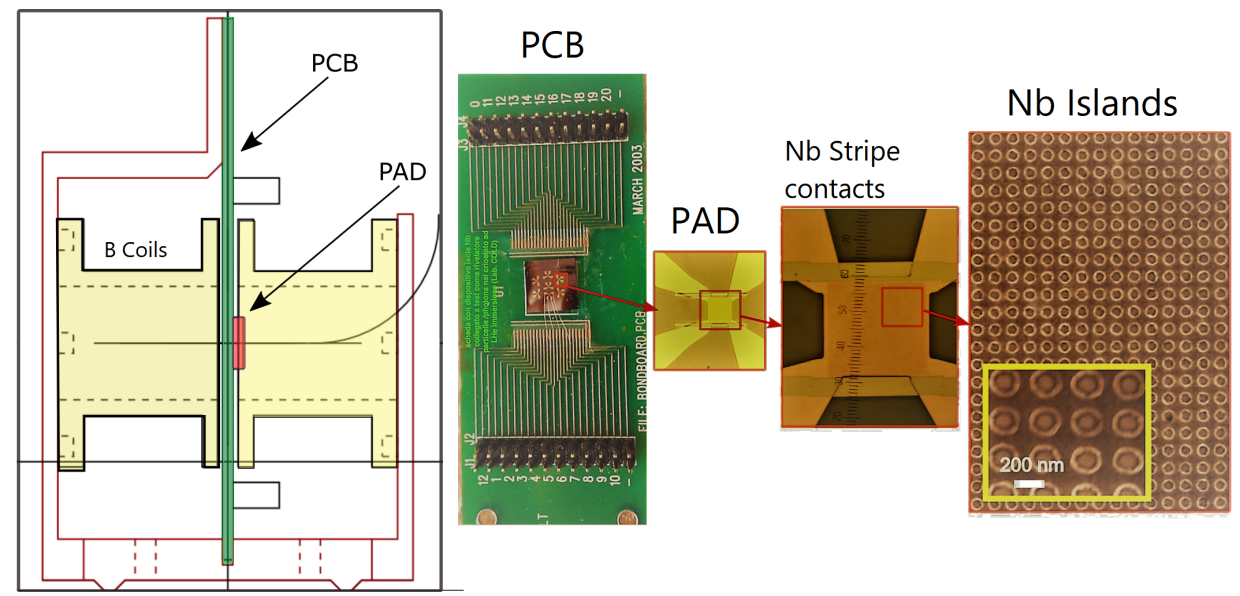

Figure 1. The sample positioning within the cryostat between the magnetic coils. The device was mounted on a PCB (green) connected to the electrical measurement apparatus. The enlarged images of the PCB and the mounted PADs show the presence of the uniform injecting Nb stripes while the SEM images show the $\mathrm{Nb}$ islands of the device.

\section{Results and Discussion}

The transition behavior of the PAD was investigated while cooling to the liquid helium temperature and is shown in Figure 2. The experimental curve points out a percolative transition around the critical temperature $\mathrm{T}_{\mathcal{c}}$. It occurs via a multistage coherence development by decreasing the temperature. At $T \geq T_{1}$, grains of each islands are in an incoherent non-superconducting phase (i.e., the normal state). As they are poly-crystalline islands, initially $\left(T \leq T_{1}\right)$ a coherent phase is established within island grains, after which the system resistance decreases. Lowering the temperature, the intra-island phase coherence continuously strengthens, and the resistance of the system similarly decreases rather than steeply dropping at $T_{1}$. Within this temperature domain the normal-metal coherence length increases until it becomes comparable to the spacing among islands. Then, inter-island phase coherence begins to emerge and finally, reaching $T_{2}$, the system undergoes the transition to a fully superconducting state [24-26]. In our device we could observe a high temperature limit $\mathrm{T}_{1}$ between $15-8 \mathrm{~K}$ with $\mathrm{T}_{\mathrm{C}}=7.5 \mathrm{~K}$ and a low fully coherent limit at $\mathrm{T}_{2}=4.2 \mathrm{~K}$.

The dynamics resistance measurement of the device was performed at $4.2 \mathrm{~K}$ in the fully intra-islands coherent phase as a function of the applied magnetics and of the electric field (see Figure 3). The dynamic resistance exhibits a clear evolution from the minima to maxima at the specific applied magnetic fields (frustration fields). The conversion occurs specifically at integer and fractional frustration fields $f$ being the $B / B_{0}$ with $B_{0}=27.8 \mathrm{mT}$, the ratio of the magnetic quantum flux on the area of the junction $\left(\Phi_{0} / a^{2}\right)$. The presence of minima in the dynamic resistance (and its analogous counterpart in the resistance) and singularities in the magnetization, reflects the modulation of the ground state energy due to the formation of a periodic vortex pattern, which have been previously observed [27]. However, in our experiment the conversion from minima to maxima in the dynamic resistance increasing the electric field intensity is the direct consequence of the phase transformation from a vortex Mott insulator into a metallic state, a mechanism previously observed in similar systems [28]. These results indicate the possibility to tune the vortex dynamic and to modulate the energy states with an external fields and with an enhanced response at integer and half-integer frustration factors $(f=1 / 2$ and $f=1)$ [29]. 


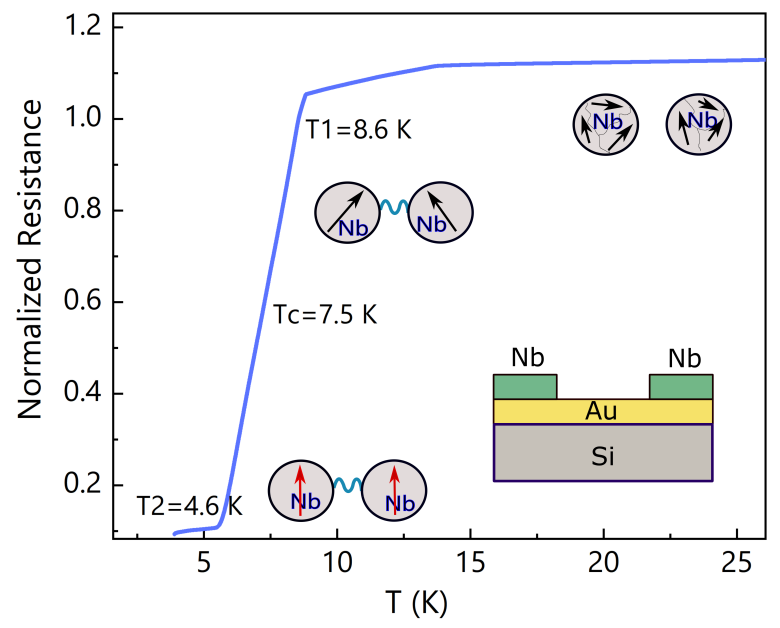

Figure 2. Percolative superconducting transition of the $\mathrm{Nb}$ islands on the Au layer. The island transition occurs at $T_{1}$ while the transition of the film is at $T_{2}$. Above $T_{1}$, the $\mathrm{Nb}$ islands behave as normal metals and the arrows indicate the coherence in grains and islands. Below $\mathrm{T}_{1}$ grains start to become coherent throughout each island and at $\mathrm{T}_{1}$, Cooper pairs start to diffuse from $\mathrm{Nb}$ into $\mathrm{Au}$, and the resistance decreases. Gradually the coherence length of the film becomes comparable to the spacing among islands, and the entire system crosses toward a global phase coherence. As the temperature decreases $\left(\mathrm{T}_{2}\right)$, the film undergoes a transition to a superconducting state. (Inset): The schematics of the islands on the gold surface deposited on the silicon substrate.
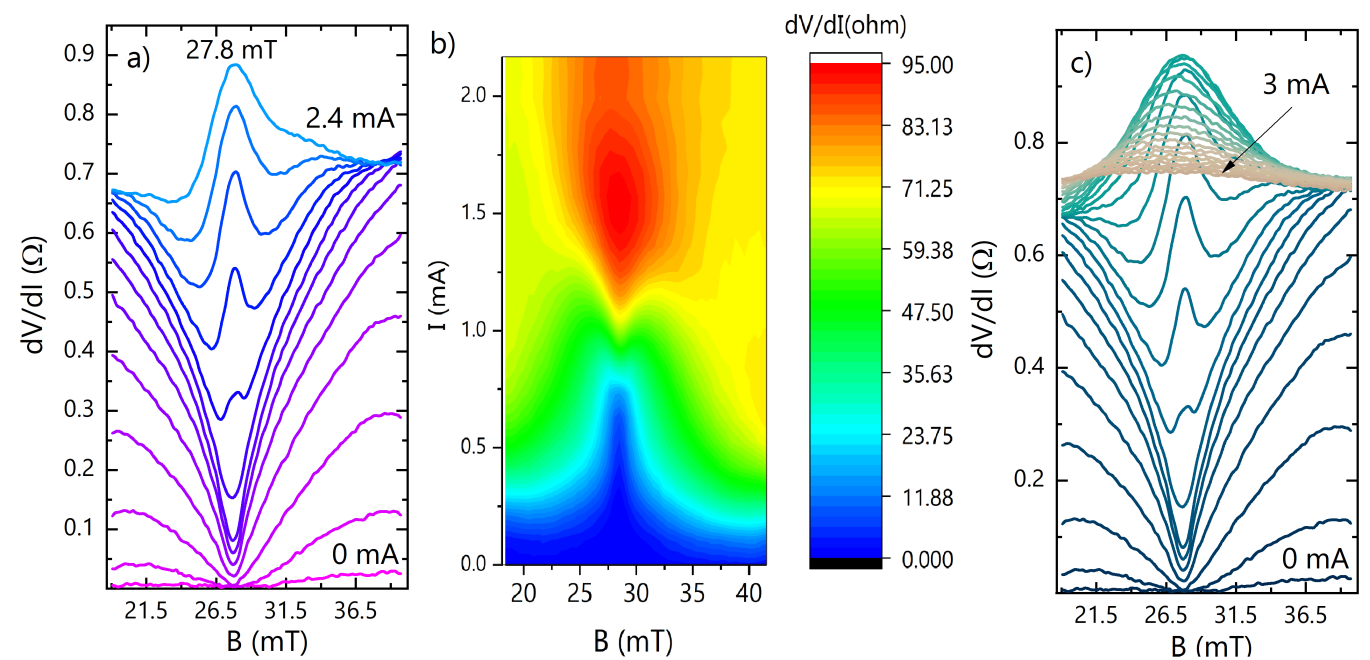

Figure 3. (a) Representative $\mathrm{dV} / \mathrm{dI}$ versus magnetic field curves at different bias currents. At low current bias, $\mathrm{dV} / \mathrm{dI}$ minima at different $\mathrm{B}$ values indicate the formation of the vortex Mott insulator. Increasing the current, minima reverse into maxima according to an insulator-to-metal transition. The transitions are most pronounced at integer and half-integer frustration factors $(f=1 / 2$ and $f=1) ;(b)$ dynamic resistance as a function of applied current and magnetic field and (c) evolution of the dynamic resistance as a function of the applied magnetic field at high applied currents; inset) the layout of the junctions formed via $\mathrm{Nb}$ islands and the Au normal layer.

The intensity increase at the integer transition points continues with the increase of the applied electric field up to a critical point of the applied current of $\mathrm{I}=2.4 \mathrm{~mA}$ at which it is severely hindered. In this region the maxima is smeared by the increase of the applied current reaching lower values if compared with the lower applied current, while the maximum also shifts toward a lower frustration value. This behavior corresponds to the emergence of a dissipative regime in which the singularities 
tends to be wiped out. However, since the resistance of our device shows pronounced dips even at large currents (not shown), indicating strong pinning, neither the minima-to-maxima transitions nor the dissipative behavior can be correlated with a vortex depinning or to the overcome of the critical current $[29,30]$. Consequently, it can be deduced that at such high current values, the metallic state features are reduced by the contribution of the quasi-particles current, occurring in the normal-metal layer [31].

The I-V measurement of the proximity junction arrays exhibits the typical Josephson behavior (see Figure 4) with the maximum tunneling current (i.e., scape current) $\mathrm{I}_{t}=1.2 \mathrm{~mA}$, over which the increase of current is accompanied by the increase of the array's potential. Above the tunneling current, the device shows a shunted behavior that might be due to either the resistive component of the device or to pairs hopping between the potential wells in a medium electric field range in which the field is not sufficient to force the junctions to the normal state. In the measurement range carried out in our experiment we did not observe the transition to the resistive normal state. Hence, the absolute value of the critical current $\left(\mathrm{I}_{C}\right)$, being relatively higher than the $\mathrm{I}_{t}$, could not be calculated. The application of the external magnetic field significantly modifies the I-V characteristics of the junction array, towards the hindrance of the junction properties (i.e., lower $\mathrm{I}_{t}$ ) due to the superimposition of the screening current generated at the edges of the superconducting contacts, to the vortex current.

On the other hand, the I-V measurements at different applied magnetic fields (see Figure 4) gradually tend to exhibit a $S$ shape behavior. This may indicate a super-relativistic motion of non-localized vortices, in which they can overcome the characteristic electromagnetic wave velocity [32]. This motion should be accompanied by Cherenkov radiation of Josephson plasma waves with a dispersion relation of the eigenmodes given by $\omega_{m}^{2}=\omega_{p}^{2}+c_{m}^{2} k^{2}$ using the Sine-Gordon equations where $c_{m}$ are the characteristic velocities [32-34]. However, in this type of proximity array the Cherenkov oscillations induced by moving fluxons are damped by the increased quasi-particle conductance. As a consequence, the decay length of the oscillating tail associated with the fluxon decreases and the resonances on the fluxon step disappear, in agreement with the observed $S$ shape behavior. The observation of the resonance steps can appear either increasing the magnetic field (below $\mathrm{H}_{c 2}$ ) or by decreasing the measurement temperature to values well below $\mathrm{T}_{2}$.
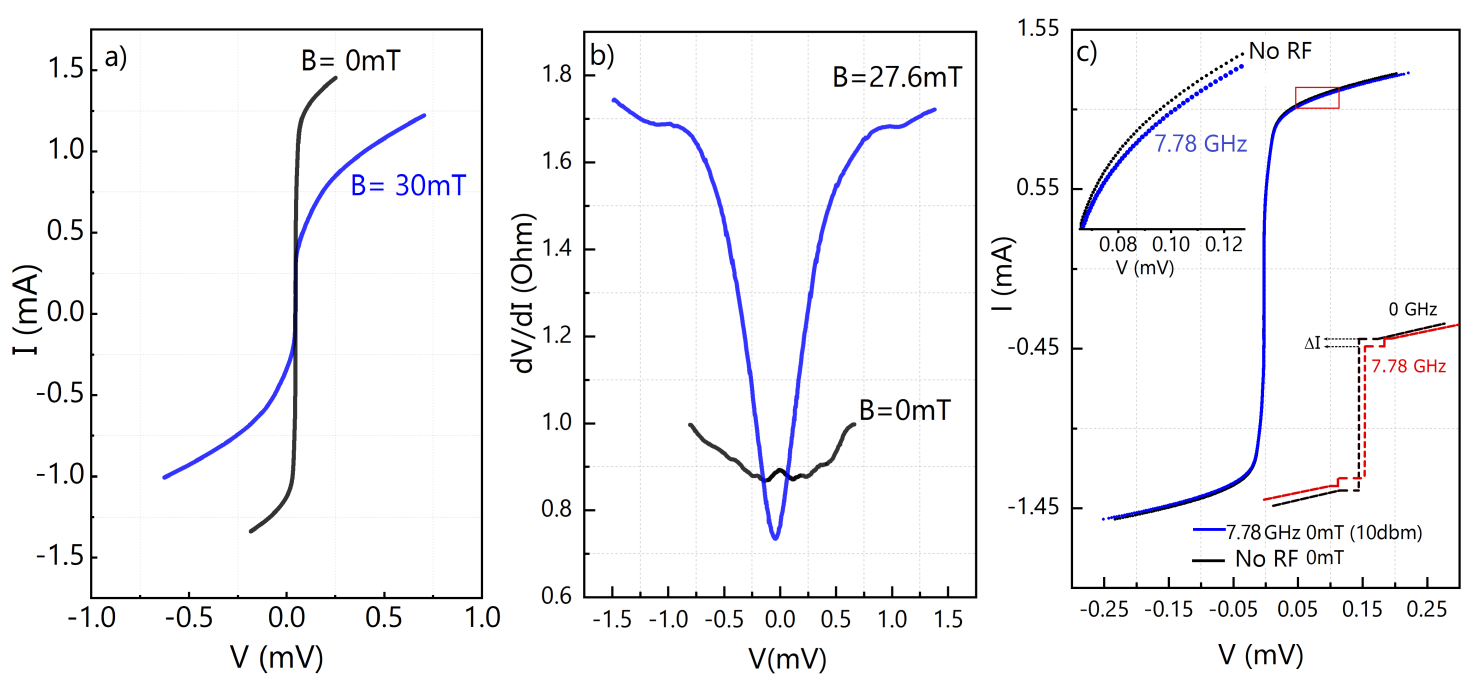

Figure 4. (a) I-V curves measured with and without the applied external magnetic field of $\mathrm{B}=27.6 \mathrm{mT}$; (b) Andreev spectra obtained from the numerical derivative of the I-V curves. The reflection peaks observable at zero field are shifted towards the higher potentials; (c) I-V response of the PAD at 7.78 GHz. The red rectangular range above $\mathrm{I}_{t}$ is expanded in the plot. The lower inset schematically shows the expected formation of the Shapiro steps and the voltage response of the device. 
The representative of the Andreev spectra (shown in Figure $4 \mathrm{~b}$ ) of our device was achieved by the numerical derivative of the I-V curves. The reflection peaks at zero field spectra are clearly visible. From them, the gap of the PAD is $\Delta \sim 1.3 \mathrm{meV}$. At the magnetic field corresponding to the frustration $f=1$, the gap peculiarities in the spectra are not only shifted towards lower energies as is expected in the classical case, but they move in the inverse direction and the intensity increases. This anomalous behavior implies that in the non-localized vortex state a triplet Cooper pairing may occur, with the total spin moment of the superconducting configuration $S=1$. In this case, in agreement with the observed vortex dynamics reported in Figure 3, a moderate magnetic field can contribute to the stabilization or even to the enhancement of the superconducting state [35] and hence to the superconducting order parameter.

The response of the device to a radio-frequency (RF) signal was measured in a non-bolometric regime in absence of applied magnetic field $(f=0)$. The response of the device to the signals with frequency lower than that of characteristic frequency (RF signal in our case) can be characterized as a perturbation to the system at non-equilibrium that results in the hindrance of the scape current $\left(\mathrm{I}_{t}\right)$ and the increase of the scape rate. This modulation directly corresponds to the RF intensity power and frequency [36]. However, the response of the device at frequencies close to the characteristic frequency (THz, in our case), should result in formation of the Shapiro steps, corresponding to the incident frequency [37].

The RF frequency was set to $7.78 \mathrm{GHz}$, the resonance frequency of the dipole antenna, though far lower than the expected characteristic frequency of the device calculated using the $I_{t}$ and the measured normal resistance of the device. The characteristic frequency was calculated considering the tunneling current maximum $\left(\mathrm{I}_{t}\right)$ and the normal resistance of the device $\left(\mathrm{R}_{n}=1.1 \Omega\right)$ as $\omega_{c}=I_{t} R_{n} / \Phi_{0}$ in which $\Phi_{0}=h / 2 e$ is the magnetic flux quantum with the value of $\omega_{c}=300 \mathrm{GHz}$ [38]. Based on the sample dimensions and the distance from the RF source, the overall RF power collected by the sample was estimated to be of the order of $1 \times 10^{-6}$ times the incident power sent to the antenna. The I-V characteristic of the device was measured with and without the RF signal superimposition and it is shown in Figure 4c. The reduction of the $\mathrm{I}_{t}$, indicating the increase of the escaping rate is clearly visible. Though, as discussed above, because of the difference between the RF frequency and the characteristic frequency and the low RF power, the formation of Shapiro steps could not be observed. Nonetheless, the $\mathrm{I}_{t}$ contraction, usually associated with the scape rate, can marks the relative response of the device (see Figure 4c-inset).

From the I-V characteristics and the power coupled into the junction we were able to estimate the device voltage sensitivity. Considering the dynamic resistance of the $\mathrm{R}_{d}=\mathrm{dV} / \mathrm{dI} \sim 0.9 \Omega$ (either via the direct ac dynamic resistance, comparable to the numerically derived dc resistance) at the device operation point, slightly above the tunneling current limit $\mathrm{I}_{t}$, we calculated the voltage modulation amplitude, $\Delta V=R_{d} \Delta I$ where $\Delta I$ is the induced current change at the operation point. Accordingly, the voltage sensitivity $\eta=\Delta V / P_{r f}$ of a non-magnetically modulated device was calculated to be of the order of $\eta \sim 7000 \mathrm{VW}^{-1}$ [39], which is relatively high compared with the semiconductor detector counterpart, with sensitivities of the order of $\eta \sim 3000 \mathrm{VW}^{-1}$ [40]. However, considering the possible impedance mismatch between the antenna and the junction, further reflections could be present and an optimized signal-coupling could increase the precision of the measurements. Since the dynamic resistance of the device is subject to the modulation via the external magnetic field, it is expected that at a certain operation bias, the device response can be significantly enhanced with the application of the magnetic field [41]. Furthermore, because of its characteristic device frequency, an enhanced response in the $\mathrm{THz}$ domain is expected.

\section{Conclusions}

In this report, we present a proximity junction arrays device (PAD), as a novel long-wavelength radiation detector. We characterized the vortex dynamics of this proximity array device (PAD) vs. external parameters, e.g., magnetic and electric field, showing the occurrence of the transition from a 
Mott insulating to a metallic state. We also demonstrated that the application of the magnetic field can modulate and significantly enhance the superconducting order parameter. The device shows a shunted collective Josephson characteristics with the characteristic frequency of $\omega=300 \mathrm{GHz}$. The application of the magnetic field also introduces a superimposition of the screening current to the tunneling current, which determines its reduction. The response of the device was experimentally measured at the frequency of $f=7.78 \mathrm{GHz}$ immersed in liquid $\mathrm{He}$ at the temperature of $\mathrm{T}=4.2 \mathrm{~K}$. As a detector, this device shows a relatively high voltage sensitivity of $7000 \mathrm{VW}^{-1}$, in absence of magnetic field modulations. Our results suggest that photo-response and voltage sensitivity of the proximity junctions array, can be additionally enhanced via external parameters, i.e., geometry and magnetic field at specific operation bias.

Author Contributions: S.J.R., D.D.G., C.G., N.P., S.L., M.C.G. and A.M. designed the experiment. S.J.R., D.D.G., C.L., M.F., S.C. and C.G. performed the experiment. S.J.R., D.D.G. and C.G. analyzed the data. All authors have read and agreed to the published version of the manuscript.

Funding: This project was financially supported by project TERA, an internal call of group $\mathrm{V}^{\text {th }}$ of INFN at LNF.

Acknowledgments: S.J.R. would like to thank Antonio Grilli and Agostino Raco for their valuable technical support. We acknowledge the financial support of the Bilateral Cooperation Agreement between Italy and Japan of the Italian Ministry of Foreign Affairs and of the International Cooperation (MAECI) in the framework of the project of major relevance N. PGR0072.

Conflicts of Interest: The authors declare no conflict of interest.

\section{References}

1. Dhillon, S.S.; Vitiello, M.S.; Linfield, E.H.; Davies, A.G.; Hoffmann, M.C.; Booske, J.; Paoloni, C.; Gensch, M.; Weightman, P.; Williams, G.P.; et al. The 2017 terahertz science and technology roadmap. J. Phys. D. Appl. Phys. 2017, 50. [CrossRef]

2. Ortolani, M.; Lupi, S.; Baldassarre, L.; Schade, U.; Calvani, P.; Takano, Y.; Nagao, M.; Takenouchi, T.; Kawarada, H. Low-Energy Electrodynamics of Superconducting Diamond. Phys. Rev. Lett. 2006, 97, 097002. [CrossRef] [PubMed]

3. Nanni, E.A.; Huang, W.R.; Hong, K.H.; Ravi, K.; Fallahi, A.; Moriena, G.; Dwayne Miller, R.J.; Kärtner, F.X. Terahertz-driven linear electron acceleration. Nat. Commun. 2015, 6, 8486, [CrossRef] [PubMed]

4. Jepsen, P.; Cooke, D.; Koch, M. Terahertz spectroscopy and imaging - Modern techniques and applications. Laser Photon. Rev. 2011, 5, 124-166. [CrossRef]

5. Saeedkia, D. Handbook of Terahertz Technology for Imaging, Sensing and Communications; Woodhead Publishing: Sawston, UK, 2013.

6. Karasik, B.S.; Sergeev, A.V.; Prober, D.E. Nanobolometers for THz Photon Detection. IEEE Trans. Terahertz Sci. Technol. 2011, 1, 97-111. [CrossRef]

7. Rezvani, S.; Perali, A.; Fretto, M.; De Leo, N.; Flammia, L.; Milošević, M.; Nannarone, S.; Pinto, N. Substrate-Induced Proximity Effect in Superconducting Niobium Nanofilms. Conden. Matter 2018, 4, 1. [CrossRef]

8. Toma, A.; Tuccio, S.; Prato, M.; De Donato, F.; Perucchi, A.; Di Pietro, P.; Marras, S.; Liberale, C.; Proietti Zaccaria, R.; De Angelis, F.; et al. Squeezing Terahertz Light into Nanovolumes: Nanoantenna Enhanced Terahertz Spectroscopy (NETS) of Semiconductor Quantum Dots. Nano Lett. 2015, 15, 386-391. [CrossRef]

9. D’Apuzzo, F.; Piacenti, A.R.; Giorgianni, F.; Autore, M.; Guidi, M.C.; Marcelli, A.; Schade, U.; Ito, Y.; Chen, M.; Lupi, S. Terahertz and mid-infrared plasmons in three-dimensional nanoporous graphene. Nat. Commun. 2017, 8, 14885. [CrossRef]

10. Richards, P.L. Bolometers for infrared and millimeter waves. J. Appl. Phys. 1994, 76, 1-24, [CrossRef]

11. Ozyuzer, L.; Koshelev, A.E.; Kurter, C.; Gopalsami, N.; Li, Q.; Tachiki, M.; Kadowaki, K.; Yamamoto, T.; Minami, H.; Yamaguchi, H.; et al. Emission of Coherent THz Radiation from Superconductors. Science 2007, 318, 1291-1293. [CrossRef]

12. Du, J.; Smart, K.; Li, L.; Leslie, K.E.; Hanham, S.M.; Wang, D.H.C.; Foley, C.P.; Ji, F.; Li, X.D.; Zeng, D.Z. A cryogen-free HTS Josephson junction detector for terahertz imaging. Supercond. Sci. Technol. 2015, 28, 084001. [CrossRef] 
13. Hammar, A.; Cherednichenko, S.; Bevilacqua, S.; Drakinskiy, V.; Stake, J. Terahertz Direct Detection in YBa2Cu3O Microbolometers. IEEE Trans. Terahertz Sci. Technol. 2011, 1, 390-394. [CrossRef]

14. Seliverstov, S.; Maslennikov, S.; Ryabchun, S.; Finkel, M.; Klapwijk, T.M.; Kaurova, N.; Vachtomin, Y.; Smirnov, K.; Voronov, B.; Goltsman, G. Fast and sensitive terahertz direct detector based on superconducting antenna-coupled hot electron bolometer. IEEE Trans. Appl. Supercond. 2015. [CrossRef]

15. Carturan, S.; Maggioni, G.; Rezvani, S.; Gunnella, R.; Pinto, N.; Gelain, M.; Napoli, D. Wet chemical treatments of high purity Ge crystals for $\gamma$-ray detectors: Surface structure, passivation capabilities and air stability. Mater. Chem. Phys. 2015, 161, 116-122. [CrossRef]

16. Sartale, S.; Ansari, A.; Rezvani, S.J. Influence of Ti film thickness and oxidation temperature on TiO2 thin film formation via thermal oxidation of sputtered Ti film. Mater. Sci. Semicond. Proc. 2013, 16, 2005-2012. [CrossRef]

17. Rezvani, S.J.; Pinto, N.; Boarino, L.; Celegato, F.; Favre, L.; Berbezier, I. Diffusion induced effects on geometry of Ge nanowires. Nanoscale 2014, 6, 7469-7473. [CrossRef]

18. Rezvani, S.J.; Pinto, N.; Enrico, E.; D'Ortenzi, L.; Chiodoni, A.; Boarino, L. Thermally activated tunneling in porous silicon nanowires with embedded Si quantum dots. J. Phys. D Appl. Phys. 2016, 49, 105104. [CrossRef]

19. Pinto, N.; Rezvani, S.J.; Favre, L.; Berbezier, I.; Fretto, M.; Boarino, L. Geometrically induced electron-electron interaction in semiconductor nanowires. Appl. Phys. Lett. 2016, 109, 123101, [CrossRef]

20. Rezvani, S.; Pasqualini, M.; Witkowska, A.; Gunnella, R.; Birrozzi, A.; Minicucci, M.; Rajantie, H.; Copley, M.; Nobili, F.; Di Cicco, A. Binder-induced surface structure evolution effects on Li-ion battery performance. Appl. Surface Sci. 2018, 435, 1029-1036. [CrossRef]

21. Luukanen, A.; Hadfield, R.H.; Miller, A.J.; Grossman, E.N. A superconducting antenna-coupled microbolometer for $\mathrm{THz}$ applications. In Proceedings of the Terahertz for Military and Security Applications II, Orlando, FL, USA, 8 September 2004.

22. Ariyoshi, S.; Otani, C.; Dobroiu, A.; Sato, H.; Kawase, K.; Shimizu, H.M.; Taino, T.; Matsuo, H. Terahertz imaging with a direct detector based on superconducting tunnel junctions. Appl. Phys. Lett. 2006, 88, 203503, [CrossRef]

23. Kislinskii, Y.; Constantinian, K.; Borisenko, I.; Ovsyannikov, G.; Yagoubov, P. Submillimeter wave signal detection by bicrystal YBCO Josephson junctions at liquid nitrogen temperatures. Physica C 2002, 372-376, 436-439. [CrossRef]

24. Resnick, D.J.; Garland, J.C.; Boyd, J.T.; Shoemaker, S.; Newrock, R.S. Kosterlitz-Thouless Transition in Proximity-Coupled Superconducting Arrays. Phys. Rev. Lett. 1981, 47, 1542-1545. [CrossRef]

25. Abraham, D.W.; Lobb, C.J.; Tinkham, M.; Klapwijk, T.M. Resistive transition in two-dimensional arrays of superconducting weak links. Phys. Rev. B 1982, 26, 5268-5271. [CrossRef]

26. Pinto, N.; Rezvani, S.J.; Perali, A.; Flammia, L.; Milošević, M.V.; Fretto, M.; Cassiago, C.; De Leo, N. Dimensional crossover and incipient quantum size effects in superconducting niobium nanofilms. Sci. Rep. 2018, 8, 4710. [CrossRef]

27. Baturina, T.I.; Vinokur, V.M.; Mironov, A.Y.; Chtchelkatchev, N.M.; Nasimov, D.A.; Latyshev, A.V. Nanopattern-stimulated superconductor-insulator transition in thin TiN films. EPL 2011, 93, 47002. [CrossRef]

28. Rezvani, J.; Di Gioacchino, D.; Gatti, C.; Poccia, N.; Ligi, C.; Tocci, S.; Cestelli Guidi, M.; Cibella, S.; Lupi, S.; Marcelli, A. Tunable Vortex Dynamics in Proximity Junction Arrays: A Possible Accurate and Sensitive 2D THz Detector. Acta Phys. Polonica A 2020, 137, 17-20, [CrossRef]

29. Poccia, N.; Baturina, T.I.; Coneri, F.; Molenaar, C.G.; Wang, X.R.; Bianconi, G.; Brinkman, A.; Hilgenkamp, H.; Golubov, A.A.; Vinokur, V.M. Critical behavior at a dynamic vortex insulator-to-metal transition. Science 2015, 349, 1202-1205. [CrossRef]

30. Benz, S.P.; Rzchowski, M.S.; Tinkham, M.; Lobb, C.J. Critical currents in frustrated two-dimensional Josephson arrays. Phys. Rev. B 1990, 42, 6165-6171. [CrossRef]

31. Argaman, N. Nonequilibrium Josephson-like effects in wide mesoscopic SNS junctions. Superlattices Microstruct. 1999, 25, $861-875$. [CrossRef]

32. Zitzmann, J.; Ustinov, A.V.; Levitchev, M.; Sakai, S. Super-relativistic fluxon in a Josephson multilayer: Experiment and simulation. Phys. Rev. B 2002, 66, 064527. [CrossRef] 
33. Sakai, S.; Bodin, P.; Pedersen, N.F. Fluxons in thin-film superconductor-insulator superlattices. J. Appl. Phys. 1993, 73, 2411-2418. [CrossRef]

34. Goldobin, E.; Wallraff, A.; Thyssen, N.; Ustinov, A. Cherenkov radiation in coupled long Josephson junctions. Phys. Rev. B 1998, 57. [CrossRef]

35. Tran, V.H.; Zaleski, T.A.; Bukowski, Z.; Tran, L.M.; Zaleski, A.J. Tuning superconductivity in $\mathrm{Eu}\left(\mathrm{Fe}_{0.81} \mathrm{Co}_{0.19}\right)_{2} \mathrm{As}_{2}$ with magnetic fields. Phys. Rev. B 2012, 85, 052502. [CrossRef]

36. Schöndorf, M.; Govia, L.C.G.; Vavilov, M.G.; McDermott, R.; Wilhelm, F.K. Optimizing microwave photodetection: input-output theory. Quantum Sci. Technol. 2018, 3, 024009, [CrossRef]

37. Shapiro, S. Josephson Currents in Superconducting Tunneling: The Effect of Microwaves and Other Observations. Phys. Rev. Lett. 1963, 11, 80-82. [CrossRef]

38. Tachiki, M.; Koyama, T.; Takahashi, S. Electromagnetic phenomena related to a low-frequency plasma in cuprate superconductors. Phys. Rev. B 1994, 50, 7065-7084. [CrossRef] [PubMed]

39. Barone, A.; Paternò, G. Physics and Applications of the Josephson Effect; Wiley: New York, NY, USA, 1982. [CrossRef]

40. Jeon, Y.; Jung, S.; Jin, H.; Mo, K.; Kim, K.R.; Park, W.K.; Han, S.T.; Park, K. Highly-Sensitive Thin Film THz Detector Based on Edge Metal-Semiconductor-Metal Junction. Sci. Rep. 2017, 7, 16830. [CrossRef] [PubMed]

41. Du, J.; Hellicar, A.D.; Li, L.; Hanham, S.M.; Nikolic, N.; Macfarlane, J.C.; Leslie, K.E. Terahertz imaging using a high-Tcsuperconducting Josephson junction detector. Supercond. Sci. Technol. 2008, 21, 125025. [CrossRef]

(C) 2020 by the authors. Licensee MDPI, Basel, Switzerland. This article is an open access article distributed under the terms and conditions of the Creative Commons Attribution (CC BY) license (http://creativecommons.org/licenses/by/4.0/). 